\title{
Erratum to: Electro-Chemo-Mechanics of Solids
}

\author{
Sean R. Bishop, Nicola H. Perry, Dario Marrocchelli \\ and Brian W. Sheldon
}

\section{Erratum to:}

S.R. Bishop et al. (eds.), Electro-Chemo-Mechanics of Solids, Electronic Materials: Science \& Technology, DOI 10.1007/978-3-319-51407-9

In Copyright page, the first affiliation of Nicola H. Perry was wrongly included as "Kyushu University, Kyushu, Japan", which has now been corrected to read as "WPI-12CNER, Kyushu University, Fukuoka, Japan" and the second affiliation "Massachusetts Institute of Technology, Cambridge, MA, USA" of Nicola H. Perry was not provided earlier, has now been included. The same has been updated at the conclusion of the Preface page too. 